\title{
Substrates for pollution control in constructed wetland systems
}

\author{
Chaowen Qian ${ }^{1, a}$, Zhenling $\mathrm{Li}^{2, \mathrm{~b}}$, Yanli Ding ${ }^{3, \mathrm{c} *}$, Shaoyuan Bai ${ }^{1, \mathrm{~d}}$, ShaoBo $\mathrm{Xi}^{1, \mathrm{e}}$ \\ ${ }^{1}$ Guangxi Key Laboratory of Environmental Pollution Control Theory and Technology, Guilin \\ University of Technology, Guilin 541004, China; \\ ${ }^{2}$ Collaborative Innovation Center for Water Pollution Control and Water Safety in Karst Area, Guilin \\ University of Technology, Guilin 541004, China; \\ ${ }^{3}$ College of Earth Sciences, Guilin University of Technology, Guilin 541004, China; \\ a18593275626@163.com, 'LZLJIUJIANG@163.com, 'dyl@glut.edu.cn(Corresponding author), \\ dbaisy@glut.edu.cn, ${ }^{\mathrm{e}} 1179801951 @ q q . c o m$
}

Key words: constructed wetland, substrates, pollution control

Abstract: Substrates play important role in constructed wetland for pollutant removal, selecting suitable substrates is one of the effective ways to improve the purification performance and to prolong its service life. This paper reviews the research of constructed wetland substrates in recent years, including the decontamination effect of different substrates, removal mechanism, and the mechanism of clogging and prevents methods related with substrate. At last, the principles for substrate selection are discussed.

\section{Introduction}

Constructed wetland (CW) is a simple, inexpensive and effective wastewater purification technology with ecological functions; the research and application of $\mathrm{CW}$ have been widely developed in recent years [1]. The basic component of $\mathrm{CW}$ included substrates, plants and microorganisms. Wastewater purification in $\mathrm{CW}$ is coordinated by physical, chemical and biochemical processes [2].

Substrates are used as plant growth carrier to provide large amounts of adhesive interface for microorganisms to ensure the physical, chemical and biological reactions in $\mathrm{CW}$ for pollutant removal [3]. The choice of substrates varies depending on the type of CW. Free surface flow wetland adopts natural soil as the substrates, while the substrates choice in horizontal and vertical flow wetland tends to be diversity. However, it will take into consideration the fact of convenience and application. In general, processing is characterized by TSS, BOD and COD removal, according to the hydraulic, retention time, and the required effluent water quality, fine sand, coarse sand, gravel, ash in one or two could be selected as the substrates. For the purpose of phosphorus removal, calcite, marble or $\mathrm{Ca}_{2}{ }^{+}, \mathrm{Fe}_{2}{ }^{+}, \mathrm{Al}_{3}{ }^{+}$ion more ore are recommended [4-5].

The substrates purifying function mainly includes: (1) the absorption and transformation of green plant roots, degradation, and biosynthesis of action; (2) substrates such as bacteria, fungi and actinomycetes in the immobilized microbial degradation, transformation and biological role; (3)The substrates of organic, inorganic gel substrates and its absorption, complexion and complex precipitation; (4)Substrates of the ion exchange; (5) The mechanical resistance of substrates and plants leave;(6)The gas diffusion of substrates[6].

Besides the effect for pollutant removal, substrates are also maintained the hydraulic conductivity performance of CW [7]. Particle size of the substrates, should not only has specific surface area for the microbes to provide more attached to medium, and can guarantee a certain 
hydraulic conductivity performance, prevent the bed body quickly blocked.

\section{Substrates for decontamination mechanisms and influence factors}

Substrates for nitrogen removal. In CW, nitrogen could be removed in several ways, including volatile, ammonification, sequential nitrification/denitrification, plant extraction and substrate adsorption. Among them, nitrification/denitrification is considered to be the main way for nitrogen removal [8]. Nitrification may be only change the form of nitrogen, only denitrification can make nitrogen into $\mathrm{N}_{2}$ and $\mathrm{N}_{2} \mathrm{O}$ from the system. Therefore, $\mathrm{CW}$ has better performance for nitrogen removal than the activated sludge system without the denitrification step, and saves much more than $\mathrm{A} / \mathrm{A} / 0$ constructed systems [1].

The reasonable adopted matrix can contribute to the high removal efficiency of nitrogen. Li Qionghui, et al. to investigate the 6 kinds of constructed wetland padding on the removal efficiency of ammonia nitrogen pollution in wastewater, through the static shaking table experiment, found that zeolite for ammonia nitrogen removal capability is best; removal rate can reach $87.65 \%$ [9]. Wu Xuanyun use of 6 kinds of substrates, such as different combination of ammonia nitrogen removal in wastewater, found that the combination of the zeolite and soil can make the water ammonia nitrogen level of the average content of relevant standard [10]. However, it should be noticed that the substrates of ammonia nitrogen adsorption are often thought to be rapidly reversible [11].

All the physical and chemical characters of CW are likely to influence the effect of removing nitrogen. The particle size and particle size distribution will affect the sewage flow velocity and water contact with the substrates in the wetland area. Larger size of substrates can effectively prevent the occurrence of clogging, but will shorten the hydraulic retention time, particle size influence purification effect, porosity as large as possible means can hold more water, and make the sewage within the wetland by adsorption and absorption for a long time [12]. Therefore, to ensure the purification effect, it is necessary to find a balance between particle sizes and adsorption capabilities [13].

Substrates for phosphorus removal. Substrates affected phosphorus removal including packing the absorption and filtering of physical chemistry. When the sewage flow through the CW, substrates remove the nitrogen, phosphorus and other nutrients in the water by some physical and chemical methods such as absorption, adsorption, filtration, ion exchange, winding and reaction, et.al.[14]. Substrates for phosphorus removal in CW include two processes, physical removal and chemical precipitation. Physical adsorption refers to the phosphorus through wetland substrates' filtration and deposition process. While chemical removal mainly occurs in the substrates, and is considered to be the main mechanism of the wetland phosphorus removal [15].

Zhang et al. selected 10 kinds of CW substrates for phosphorus adsorption; it was found that smaller bulk density and larger porosity were benefit for the effect of phosphorus removal [4]. Wu et al. chose three kinds of $\mathrm{CW}$ substrates for adsorption isotherm and adsorption kinetics experiments, found that anthracite was the most effective substrate for phosphorus removal and the removal rate reached $96.48 \%$ to $99.19 \%$, respectively [16]. Zhong et al. compared 7 kinds of substrates for phosphorus removal. It was found that phosphorus removal rates of steel slag, coal cinder and oyster shells were increased with the initial phosphorus concentration and decreased material particle size, Among them, the steel slag removal effect is best ${ }^{[14]}$.

\section{The substrates affected clogging and prevent methods}

Clogging is a complex process during $\mathrm{CW}$ operation. Du et al. divided constructed wetland 
substrate clogging occurring into three stages: The first stage, the penetration rate is slow down, and the falling rate is not obvious; The second phase, the matrix permeability rate substantial dropped steadily; The third stage, constructed wetland substrate intermittent system congestion phase until last clogging occur and harmony on the surface of the water, the constructed wetland in anaerobic condition [17]. For horizontal undercurrent of CW clogging, Chen reported it may be related to the large particles of local siltation, suspended solids in water and fall off of biofilm packing surface deposition [18].

The matters caused CW clogging can be roughly divided into three kinds: organic blockage, suspended solid blockage and gas blockage. The organic matter block is the main reason. Organic loading has a great effect for clogging, especially for the organic suspended solids loadings. Besides that, plants in wetland are also in high productivity and high potential organic load for the adequate nutrition in wastewater [19].

\section{Problems and Prospect}

Research about CW has been developed rapidly in recent decades, however, in the actual application process, there are still a lot of technical bottleneck problems need to be solved. First of all, after running a period of time, substrates of $\mathrm{CW}$ tend to clogging due to organic matter accumulation. This will directly affect the running effect of wetlands; especially for reducing the removal performances of nitrogen and phosphorus in wastewater. Clogging is always a difficult problem for wetland running. There are several ways to alleviate clogging, choose the right size and grading, pretreatment the water, reasonable water distribution way, appropriate wetland plant and regularly replace the wetland surface substrates [6].

The second problem is adsorption saturation. Substrates will reach saturation when it runs several years or remove mass of pollutants. Once the substrates saturated, it will lost decontamination effect, and may also release some pollutants then cause second pollution. Therefore, saturated time determines the running life of the constructed wetland. How to use efficient substrates and how to extend saturated time or to renew the saturated substrates are also the main difficulties for the present study [8].

At last, researches about substrates effects usually depend on $2 \sim 4$ kinds of substrates, or only for a certain type of pollutant components (organic matter, nitrogen, phosphorus, et al. which lead to the result limitations. Widely collect different types and different quality of material, different regions of substrate is necessary to complete all kinds of pollutants removal analysis, for the removal effect of different substrates and substrates composition changes before and after the adsorption experiments [20].

\section{Acknowledgements}

This work was funded by the National Natural Science Foundation of China (No. 51408147, 41404116, 51638006), Science Research and Technology Development Project of Guangxi ( Guikehe1599005-2-2) and was supported by the Guangxi Scientific Experiment Center of Mining, Metallurgy and Environment ( KH2012ZD004) and the project of high level innovation team and standing scholar in Guangxi colleges and universities ( 002401013001 ) 


\section{Reference}

[1] Xiaolei Wu. Environmental science.1995. Forum Vol.03 (1995): 83-84.In Chinese.

[2] Jingjing Ruan, De Gao, Jamming Hong, et al. Journal of Capital Normal University. Forum Vol.12 (2009): 85-90.In Chinese.

[3] Zixia Li, Xiaodan Tian, Lihua Cui, et al. Chinese Journal of Environmental Engineering. Forum Vol. 2(2016):638-642.In Chinese.

[4] Xiuwen Zhang, Fengmin Li, Lun Lu, et al. TECHNOLOGY OF WATER TREATMENT. Forum Vol. 3(2014):49-56.In Chinese.

[5] Bo Liu. Chinese Journal of Environment Engineering. Forum Vol.1 (2010):44-48.In Chinese.

[6] Jiajing Wan, Zhan Wang, Jun Li, et al. Environmental protection science. Forum Vol. 6(2009):16-19. In Chinese.

[7] Hucheng Zhang, Wei Tian, Muqing Yu, et al. Techniques and Equipment for Environmental Pollution Control. Forum Vol.2 (2004):11-15. In Chinese.

[8] Lingxiang Zhang. Municipal Engineering Technology. Forum Vol.5 (2008):213-216. In Chinese.

[9] Qionghui Li, Weipeng Yu, Xiaorong Li, et al. Journal of Anhui Agri Sci Forum Vol. 44(2016): 83 - 86. In Chinese.

[10] Xuanyun Wu, Xiangyang Sun, Yaning Luan, et al. ECHNOLOGY OF WATER TREATMENT. Forum Vol.2 (2015):52-55. In Chinese.

[11] Shaoyong Lu, Xiangcan Jin, Gang Yu. ACTA ECOLOG ICA SINICA. Forum Vol. 8(2006):2670-2677. In Chinese.

[12] Yi Ding, Xinshan Song, and Denghua Yan. Environmental Science \& Technology. Forum Vol. 12(2011):103-106. In Chinese.

[13] Tao Yu, Zhenbin Wu, Dong Xu, et al. Environmental Science \& Technology. Forum Vol. 6(2009):74-77. In Chinese.

[14] Zongqiang Zhong. Communicate with case of scientific papers. Forum Vol. 11(2015):66-71. In Chinese.

[15] Guodong JI, Tieheng Sun, Shun Li. CHINESE JOURNAL OF APPLIED ECOLOGY. Forum Vol. 13(2002):224-228.In Chinese.

[16] Xuanyun $\mathrm{Wu}$, Yaning Luan, Xiaoqiang Gong, et al. Chinese Journal of Environmental Engineering. Forum Vol. 1(2015):257-263. In Chinese.

[17] Zhongdian Du, Lihua Cui, Zhen Xiao, et al. Argo-environment protection. Forum Vol. 21(2002):474-476. In Chinese.

[18] Xiaodong Chen. Environmental protection science. Forum Vol. 10(2011):19-22. In Chinese.

[19] Jie Zhu, Hongbin Chen. CHINAWATER \& WASTEWATER. Forum Vol. 3(2009):24-33. In Chinese.

[20] Wenxi Zhao, Lei Tao, Honglei Liu. ENVIRONMENTAL SCIENCE AND MANAGEMENT. Forum Vol. 8(2013):8-16. In Chinese. 